\title{
Efficacy and safety of new generic of direct acting antivirals in the treatment of chronic hepatitis $\mathrm{C}$
}

\author{
Fatiha Bouhamou*, Sara Morabit, Sanaa Berrag, Mouna Tazaourte, Rouibaa F and Aourarh A \\ Department of Gastroenterology, Military Hospital Mohamed V, Rabat, Morocco
}

\begin{abstract}
The management of chronic hepatitis $\mathrm{C}$ infection is currently in change. IFN based treatments were the standard therapy, against $\mathrm{HCV}$ infection waiting for pending authorization to place the direct acting antivirals on the market. These new direct acting antivirals generic conferred good effectiveness and safety for infected patients. The aim of this study was to characterize the treatment response and tolerance of new generics of DAAs in patients infected with hepatitis C virus. The study was conducted at the gastroenterology I department of the military hospital Mohamed V in Rabat since December 2015. We include all patients infected with HCV: naif relapsing or non-responsive profiles, all genotypes combined, cirrhotic or not. They all received treatment with the new Moroccan generic of direct acting antivirals. Virological response as well as clinical and biochemical tolerances were achieved. 77 patients with viral hepatitis $\mathrm{C}$ were included in the study. The average age of the patients was $61 \pm 11$ years old. A slight predominance of women noted in $52.8 \%$ of cases. Genotype 1 was predominant in $74 \%$ of cases. Half of our patients were cirrhotic. The sustained virogical response SVR was of the order of $96.2 \%$. The treatment generally well tolerated in all our patients. The generic of direct acting antivirals promises treatments with shorter treatment times, much higher cure rates, and fewer side effects. Viral eradication leads stabilization or regression of fibrosis.
\end{abstract}

\section{Introduction}

Hepatitis $\mathrm{C}$ virus (HCV) infection has a high prevalence worldwide and is the leading cause of cirrhosis and hepatocellular carcinoma $[1,2]$. The antiviral treatment for HCV infection, reduces liver disease progression and improves the quality of life of patients who obtain sustained virological response (SVR) [3,4] defined as undetectable HCV RNA 12 weeks (SVR12) or 24 weeks (SVR24) after treatment completion. Achieving an SVR is, for the vast majority of patients, synonymous with curing hepatitis $\mathrm{C}$.

Researchers have recently made significant advances in treatment for hepatitis $\mathrm{C}$ using new, "direct-acting" antiviral medications. Most of these individual drugs are effective for specific strains, or genotypes, of HCV. However, some newer combination medications, which contain two or more drugs, work for all genotypes. Most are available in pill form. Typically, these pills have far fewer side effects than previous treatment options.

However, a downside to these successful drug development efforts is their hefty cost of treatment. These high costs of innovative Hepatitis $\mathrm{C}$ treatments have led to limits in access to treatment for patients with chronic Hepatitis C.

In Morocco, we only have Sofosbuvir, Daclatasvir and Ribavirin. The other treatments are off-label and not reimbursed. The main objective of this study is to prove the efficacy and safety of the generic SOFOSBUVIR plus DACLATASVIR in patients with HCV genotypes 1 or 2 infections.

\section{Patients and methods}

\section{Patient enrollment}

Was included all patients infected with HCV: naif relapsing or non-responsive profiles, all genotypes combined, cirrhotic or not. They all received treatment with the generic of direct acting antivirals at the gastroenterology I department of the military hospital Mohamed V in Rabat, Morocco since December from December 2015 to November 2019. Chronic hepatitis C defined as the presence of HCV antibody and detectable serum HCV RNA. Patients with HIV infection, detectable hepatitis B surface antigen, evidence of other liver disease (e.g., autoimmune hepatitis and primary biliary cirrhosis), hemodialysis patients, patients with HCC and decompensated cirrhosis were excluded from the study.

Patients with chronic hepatitis $\mathrm{C}$ with compensated (Child-Pugh A) cirrhosis or without cirrhosis genotype 1a treatment naïve (defined as patients who have never been treated for their HCV infection)/G1b/ G2 received SOF DCV for 12 weeks. Patients with chronic hepatitis C with compensated (Child-Pugh A) cirrhosis or without cirrhosis genotype 1a treatment experienced (defined as patients who were previously treated with pegylated IFN-a and ribavirin] received SOF DCV for 12 weeks [3].

\section{Data collection}

Baseline data were collected from medical records. They included demographic information, HCV genotype, and liver fibrosis evaluation.

${ }^{\star}$ Correspondence to: Fatiha Bouhamou, Department of Gastroenterology, Military Hospital Mohamed V, Rabat, Morocco, Tel: 212678346021; E-mail: f.bouhamou@gmail.com

key words: virogical response, hepatitis $C$ virus, fibrosis

Received: January 09, 2020; Accepted: January 23, 2020; Published: January 27 2020 


\section{Fibrosis evaluation}

We used non-invasive methods [combination of liver stiffness measurement and a blood test] to Identified patients with cirrhosis (METAVIR score F4) or advanced (bridging) fibrosis (METAVIR score F3).

\section{Statistical analysis}

Analysis of variance (standard or nonparametric, as appropriate) was used for continuous variables whereas the chi-square test was used for categorical variables. All analyses were performed with SPSS software 20. (Statistical package for the social sciences). A significance level of $5 \%(p<0.05)$ was considered statistically significant.

\section{Results}

\section{Pretreatment demographic and clinical characteristics}

A total of 77 patients were included in the study. Patients' characteristics are presented in table $1.52 .8 \%$ were women, and the median age at the beginning of follow up was 61 years. Only $26 \%$ patients were infected by HCV genotype $2.50 \%$ of patients were cirrhotic. $66.2 \%$ of patients were treatment naive and $33.8 \%$ treatment experienced.

\section{Virological response and side effects}

Of 77 patients treated for 12 or 24 weeks, 73 (96.1\%) patients had SVR and four cases has failed to achieve SVR after treatment. The most common adverse events were fatigue, headache, nausea, insomnia and one case of esophageal candidiasis (Figure 1).

\section{Discussion and conclusion}

Clinical care for patients with HCV-related liver disease has advanced considerably during the last two decades, thanks to an enhanced understanding of the pathophysiology of the disease, and because of developments in diagnostic procedures and improvements in therapy and prevention. Actually IFN-free, ribavirin-free, DAA-based regimens must be used in $\mathrm{HCV}$-infected patients without cirrhosis or

Table 1. Characteristics of 93 study patients with chronic hepatitis $\mathrm{C}$ virus infection [December 2015-November 2019].

\begin{tabular}{|c|c|}
\hline Characteristic & Value \\
\hline male-n \% & $52,8 \%$ \\
\hline age [years] & $61 \pm 11$ \\
\hline Cirrhosis -n\%, & $50 \%$, \\
\hline Treatment naive & $66.2 \%$, \\
\hline treatment experienced & $33.8 \%$, \\
\hline
\end{tabular}

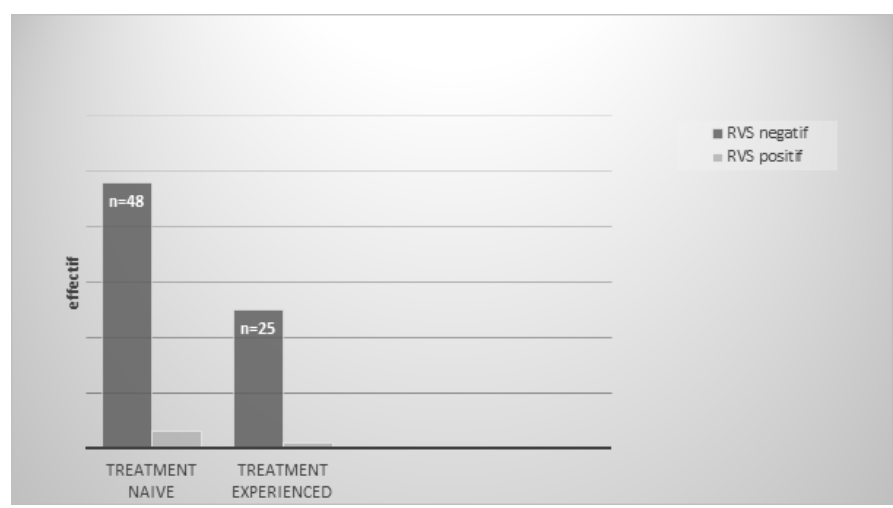

Figure 1. Sustained virologic response rate in subgroups of patients treated for HCV with compensated (Child-Pugh A) cirrhosis, including "treatmentnaïve" patients and "treatment-experienced" patients, because of their virological efficacy, ease of use, safety and tolerability [4].

In Morocco, because of the unavailability of new molecules combination Sofosbuvir/Elpatasvir. Glecaprevir/Pibrentasvir, Grazoprevir/ Elbasvir. We are using a combination of Sofosbuvir (a nucleotide analogue HCV NS5B polymerase inhibitor) administered at the dose of $400 \mathrm{mg}$ (one tablet) and Daclatasvir (an HCV NS5A replication complex inhibitor), administered at the dose of $60 \mathrm{mg}$ (one tablet).

In the present study, the rates RVS was $96.1 \%$. Overall, the rate of SVR found in this study was similar to that reported with combination of Sofosbuvir and Daclatasvir in patients without cirrhosis, Phase IIb results. Studies have shown SVR rates ranging from $92 \%$ to $98 \%$, and in the current study, the SVR rate was $96.1 \%$ [5].

In conclusion, regarding the SVR rate among the studied patients, Sofosbuvir plus Daclatasivr was better than biosimilar standard IFN for genotype 2 or 3 infections regardless of fibrosis stage, and the SVR rate associated with biosimilar standard IFN was low. Therefore, PegIFN is a better option for genotype 2 or 3 infections than biosimilar standard IFN.

In conclusion, Once-daily oral daclatasvir generic plus sofosbuvir generic was associated with high rates of sustained virologic response among patients infected with HCV genotype 1, 2, including patients with no response to prior therapy with INF regimen.

\section{References}

1. Polaris Observatory HCV Collaborators (2017) Global prevalence and genotype distribution of hepatitis C virus infection in 2015: a modelling study. Lance Gastroenterol Hepatol 2: 161-176. [Crossef]

2. European Union HCV Collaborators (2017) Hepatitis C virus prevalence and level of intervention required to achieve the WHO targets for elimination in the European Union by 2030: a modelling study. Lancet Gastroenterol Hepatol 2: 325-336. [Crossef]

3. European Association for the Study of the Liver (2017) EASL Recommendations on Treatment of Hepatitis C 2016. J Hepatol 66: 153-194. [Crossref]

4. European Association for the Study of the Liver (2018) EASL Recommendations on Treatment of Hepatitis C 2018. J Hepatol 66: 461-511. [Crossref]

5. Sulkowski MS, Gardiner DF, Rodriguez-Torres M, Reddy KR, Hassanein T, et al. (2014) Daclatasvir plus sofosbuvir for previously treated or untreated chronic HCV infection. N Engl J Med 370: 211-221. [Crossef]

Copyright: (C2020 Bouhamou F. This is an open-access article distributed under the terms of the Creative Commons Attribution License, which permits unrestricted use, distribution, and reproduction in any medium, provided the original author and source are credited. 